\title{
Thermodynamic instabilities of generalized exotic BTZ black holes
}

\author{
Wan Cong and Robert B. Mann \\ Department of Physics and Astronomy, University of Waterloo, \\ 200 University Ave W, Waterloo, Ontario, N2L 3 G1 Canada \\ Perimeter Institute for Theoretical Physics, \\ 31 Caroline St. N., Waterloo, Ontario, N2L $2 Y 5$ Canada \\ E-mail: wcong@uwaterloo.ca, rbmann@uwaterloo.ca
}

ABSTRACT: We examine the conjecture that black holes violating the reverse isoperimetric inequality have negative specific heat at constant volume $C_{V}[1]$. We test this conjecture on the family of generalized exotic Bañados, Teitelboim and Zanelli (BTZ) black holes and find that $C_{V}$ can be positive even when the reverse isoperimetric inequality is violated, providing a counter example to the conjecture. However in all cases where $C_{V}$ is positive, the specific heat at constant pressure $C_{P}$ is negative, indicating that generalized exotic black holes are thermodynamically unstable, suggesting that a broader version of the conjecture might hold.

KEYwords: Black Holes, Classical Theories of Gravity

ARXIV EPRINT: 1908.01254 


\section{Contents}

1 Introduction 1

1.1 Generalised BTZ black holes 2

2 Results 3

3 Conclusion 5

\section{Introduction}

In the framework of extended black hole thermodynamics [2-6], the cosmological constant $\Lambda$ is interpreted as a pressure variable, $P=-\frac{\Lambda}{8 \pi}$, whose variation leads to the extended first law, $d M=T d S+V d P$. Identifying the black hole mass $M$ as the enthalpy of the black hole, this looks like the usual first law of thermodynamics for systems with volume. The volume term $V$ in the extended first law is the conjugate potential to $P$, with $V=\left.\frac{\partial M}{\partial P}\right|_{S}$. However this "volume" is not simply the geometric volume of the black hole except in simple cases such as the Anti-de Sitter (AdS) Schwarzchild black hole. Indeed, one need not even have a black hole present to define this volume; a single cosmological horizon is sufficient [7].

It has remained an ongoing puzzle to better understand the physical interpretation of this volume term. An early attempt to do so [3] led to the conjecture that asymptotically AdS black holes satisfy the reverse isoperimetric inequality,

$$
\mathcal{R}=\left(\frac{(d-1) V}{\omega_{d-2}}\right)^{\frac{1}{d-1}}\left(\frac{\omega_{d-2}}{A}\right)^{\frac{1}{d-2}} \geq 1
$$

for a $d$-dimensional spacetime. In this expression, $\omega_{d-2}$ is the dimensionless surface area of the unit ball in $d-1$ dimensions and $A$ is the surface area of the black hole horizon. The conjecture was motivated by the observation at the time that all known black holes seemed to respect the inequality (1.1). When the equality is saturated, a black hole of a given thermodynamic volume has attained its maximal possible entropy.

Since then, the isoperimetric ratio $\mathcal{R}$ of various black hole families have been studied, and some were found to violate this inequality [8-16]. Such black holes have come to be called super-entropic, since they have more entropy than the relation (1.1) would admit.

What is still missing however is a physical interpretation of such violations. A recent proposal [1] hopes to remedy this shortfall by positing that violation of the reverse isoperimetric inequality implies a new type of thermodynamic instability, namely that the specific heat at constant volume, $C_{V}$, of the black hole will be negative. This was shown to be the case analytically for the $(2+1)$-dimensional charged Bañados, Teitelboim and Zanelli (BTZ) black holes [17] as well as numerically for the ultra spinning $d$-dimensional 
Kerr black hole [10]. Systems with negative specific heat are unstable - an initial loss of energy is accompanied by an increase in temperature, leading to faster energy loss and a further increase in temperature. The system thus accelerates through this downward spiral rather than settling down to a nearby equilibrium state. In the case of black holes, this loss of energy can for example be due to Hawking Radiation. Traditionally, such instabilities were studied by looking at the sign of $C_{P}$, the specific heat at constant pressure. With the introduction of the volume term in extended thermodynamics it is natural to also look at $C_{V}$, which may harbour interesting physics $[1,18]$.

Here we test the conjecture of [1] using the family of "generalized exotic BTZ black holes", a term coined in [19]. Such black holes violate the inequality (1.1) and so are superentropic. The relative simplicity of the spacetime makes them ideal candidates for studying their specific volumes and so testing the new instability conjecture. We find that generalized exotic BTZ black holes do not always have $C_{V}<0$, and so provide a counterexample to the conjecture. However we also find that whenever $C_{V}>0$ that $C_{P}<0$, indicating such black holes are in general thermodynamically unstable. This suggests a broader version of the instability conjecture: all super-entropic black holes are thermodynamically unstable.

\subsection{Generalised BTZ black holes}

A study of the thermodynamics of BTZ black holes [20] indicated that charged BTZ black holes were super-entropic. Generalized BTZ black holes are also super-entropic, but demonstration of this is somewhat more subtle. These objects are $(2+1)$-dimensional black holes whose metric is given by the usual rotating BTZ metric

$$
d s^{2}=-f(r) d t^{2}+\frac{1}{f(r)} d r^{2}+r^{2}\left(d \phi-\frac{4 j}{r^{2}} d t\right)^{2}
$$

with

$$
f(r)=-8 m+\frac{r^{2}}{\ell^{2}}+\frac{16 j^{2}}{r^{2}},
$$

where $\ell$ is the AdS radius. Here and below, we work with units in which $\hbar=G=c=$ $k_{B}=1$. The roots of the function $f(r)$ give the inner and outer black hole horizons, $r_{ \pm}=2 \sqrt{\ell\left(\ell m \pm \sqrt{\ell^{2} m^{2}-j^{2}}\right)}$. This metric is a solution to both the usual 3D Einstein action with a negative cosmological constant, as well as the gravitating Chern-Simons (GCS) action [21]. The expressions for the conserved mass $M$ and angular momentum $J$ as functions of the parameters $m$ and $j$ depend on the choice of action. In the former well-known case $m$ and $j$ are respectively the mass and angular momentum of the black hole, while for the latter action we have the "exotic" case, with [22]

$$
M=j / \ell, \quad J=\ell m
$$

Note that varying the GCS action yields the same equations of motion as the usual EinsteinHilbert (EH) action in 3D but a computation of the Noether charges (see for example [23] for an explicit computation) will reveal the reversed roles in eq. (1.4). This reversed role of the $m$ and $j$ parameters has also been found in other gravity models [24-27]. 
If one takes the gravitational action to be $I=\alpha I_{\mathrm{EH}}+\gamma I_{\mathrm{GCS}}$, which is a linear combination of the EH action and the GCS action [19, 28], then one obtains the generalized exotic BTZ black holes, for which

$$
\begin{gathered}
M=\alpha m+\gamma \frac{j}{\ell}, \\
J=\alpha j+\gamma \ell m
\end{gathered}
$$

are the conserved mass and angular momentum, with $\alpha \in(0,1)$ and $\gamma=1-\alpha$. When $\alpha=1$, we have the standard BTZ black hole while $\alpha=0$ corresponds to the exotic BTZ black hole.

The thermodynamic variables of the generalized exotic BTZ black holes are [19]

$$
\begin{aligned}
& M=\frac{\alpha\left(r_{-}^{2}+r_{+}^{2}\right)}{8 \ell^{2}}+\frac{\gamma r_{-} r_{+}}{4 \ell^{2}}, \quad J=\frac{\alpha r_{-} r_{+}}{4 \ell}+\frac{\gamma\left(r_{-}^{2}+r_{+}^{2}\right)}{8 \ell}, \\
& T=\frac{r_{+}^{2}-r_{-}^{2}}{2 \pi \ell^{2} r_{+}}, \quad \Omega=\frac{r_{-}}{r_{+} \ell}, \quad S=\frac{1}{2}\left(\pi \alpha r_{+}+\pi \gamma r_{-}\right), \\
& V=\alpha \pi r_{+}^{2}+\gamma \pi r_{-}^{2}\left(\frac{3 r_{+}}{2 r_{-}}-\frac{r_{-}}{2 r_{+}}\right) .
\end{aligned}
$$

They satisfy the first law, $d M=T d S+V d P+\Omega d J$ as well as the Smarr relation, $0=$ $T S-2 P V+\Omega J$. The ratio $\mathcal{R}$ can be readily computed to be [19]

$$
\mathcal{R}=\frac{1}{2} \sqrt{4 \alpha-2 \gamma \frac{r_{-}^{3}}{r_{+}^{3}}+6 \gamma \frac{r_{-}}{r_{+}}} .
$$

The reverse isoperimetric inequality is saturated for standard BTZ black holes $(\alpha=1)$ with $\mathcal{R}=1$. For all other $\alpha$ values, $\mathcal{R} \leq 1$ with equality if and only if $r_{-}=r_{+}$, i.e., the case of extremal black holes. Hence, the reverse isoperimetric inequality is violated for all generalized exotic BTZ black holes except for the standard BTZ and extremal cases. We thus expect these black holes to have negative specific heat at constant volume according to the conjecture in [1].

\section{Results}

We begin our study of thermodynamic stability by looking at $C_{P}=\left.T \frac{\partial S}{\partial T}\right|_{P, J}$ for generalized exotic BTZ black holes. Analytic expression for $C_{P}\left(r_{+}, P, J\right)$ can be obtained by first solving for $r_{m}$ in terms of $\left(J, P, r_{+}\right)$using eq. (1.7), and then substituting this into the expressions for temperature and entropy, eq. (1.8). We can then use the relation $\left.\frac{\partial S}{\partial T}\right|_{P, J}=$ $\left.\left.\frac{\partial S}{\partial r_{+}}\right|_{P, J} \frac{\partial r_{+}}{\partial T}\right|_{P, J}$ to find $C_{P}$.

The result is shown in figure 1 (see also [19]). The range of $r_{+}$on this figure is chosen to ensure $0 \leq r_{m} \leq r_{+}$, which translates into the condition

$$
r_{E} \equiv\left(\frac{2 J^{2}}{\pi P}\right)^{\frac{1}{4}} \leq r_{+} \leq\left(\frac{8 J^{2}}{\pi P(1-\alpha)^{2}}\right)^{\frac{1}{4}} .
$$




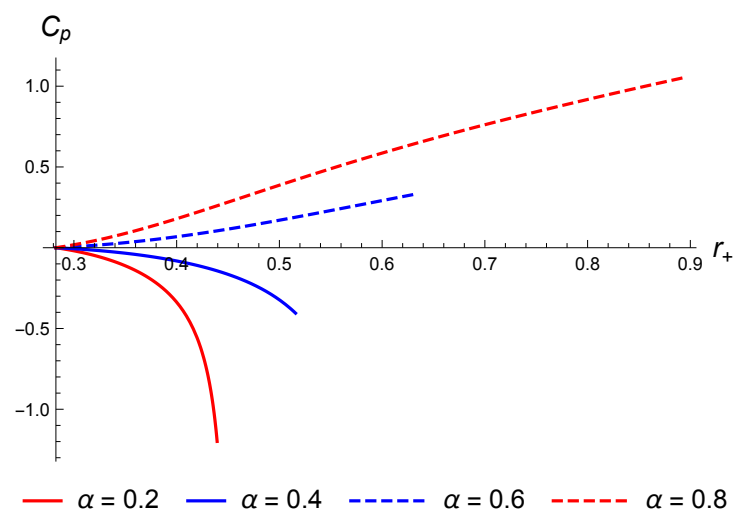

Figure 1. Plot of $C_{P}$ against $r_{+}$for various values of $\alpha$ at $P=1$ and $J=0.1$. The range of $r_{+}$in each of these plots are restricted to $\left(\frac{2 J^{2}}{\pi P}\right)^{\frac{1}{4}} \leq r_{+} \leq\left(\frac{8 J^{2}}{\pi P(1-\alpha)^{2}}\right)^{\frac{1}{4}}$ to ensure $0 \leq r_{m} \leq r_{+}$.

The black hole is extremal if $r_{+}=r_{-}=r_{E}$. For "more exotic" black holes i.e., $\alpha<1 / 2$ (solid curves) $C_{P}$ is negative except for extremal cases where it is zero while for more standard black holes $(\alpha>1 / 2) C_{P}$ is positive except again at $r_{+}=r_{E}$ where it is zero. Already, we are seeing that more exotic black holes are unstable according to the sign of $C_{P}$.

We now move on to look at $C_{V}$ for these black holes. To do this, we make use of the expression [1]

$$
C_{V}=C_{P}-T V \alpha_{T}^{2} \kappa_{T}
$$

where $\alpha_{p} \equiv V^{-1} \partial V /\left.\partial T\right|_{P, J}$ and $\kappa_{T} \equiv-V \partial P /\left.\partial V\right|_{T, J}$. Each of these expressions can be evaluated in a similar manner to $C_{P}$. In the fully exotic case we have

$$
C_{V}=\frac{-4608 S^{9}+\pi^{3} S V^{3}\left(\sqrt{48 S^{4}+\pi^{2} V^{2}}+\pi V\right)-48 \pi S^{5} V\left(\pi V-3 \sqrt{48 S^{4}+\pi^{2} V^{2}}\right)}{3\left(4608 S^{8}+48 \pi S^{4} V\left(\pi V-7 \sqrt{48 S^{4}+\pi^{2} V^{2}}\right)+\pi^{3} V^{3}\left(\sqrt{48 S^{4}+\pi^{2} V^{2}}+\pi V\right)\right)}
$$

$C_{V}$ for general values of $\alpha$ can also be computed but the resultant expressions are long and not very instructive so we exclude them here.

The plot of $C_{V}$ for various $\alpha$ values are shown in figure 2 . First, notice that $C_{V}$ of $1 / 2<\alpha<1$ (right figure) black holes are negative, in agreement with the conjecture in [1]. When $\alpha=1, C_{V}=0$, as expected for the standard BTZ black hole. Recall that the $C_{P}$ of these black holes are non-negative. This thus illustrates how the thermodynamic stability of black holes depends on whether we are keeping $P$ or $V$ fixed.

More interesting is the $\alpha<1 / 2$ (left figure) case. When $\alpha$ is close to $1 / 2, C_{V}$ remains negative, as in the red and green curves in the figure. These curves terminate at the extremal limit $r_{+}=r_{-}$. However, as the black hole becomes more exotic with decreasing $\alpha$, we see that $C_{V}$ can become positive at large enough $r_{+}$as is the case for the blue, yellow, and purple $(\alpha=0)$ curves. The divergences in $C_{V}$ occur whenever $\partial V /\left.\partial P\right|_{T, J}=0$. 


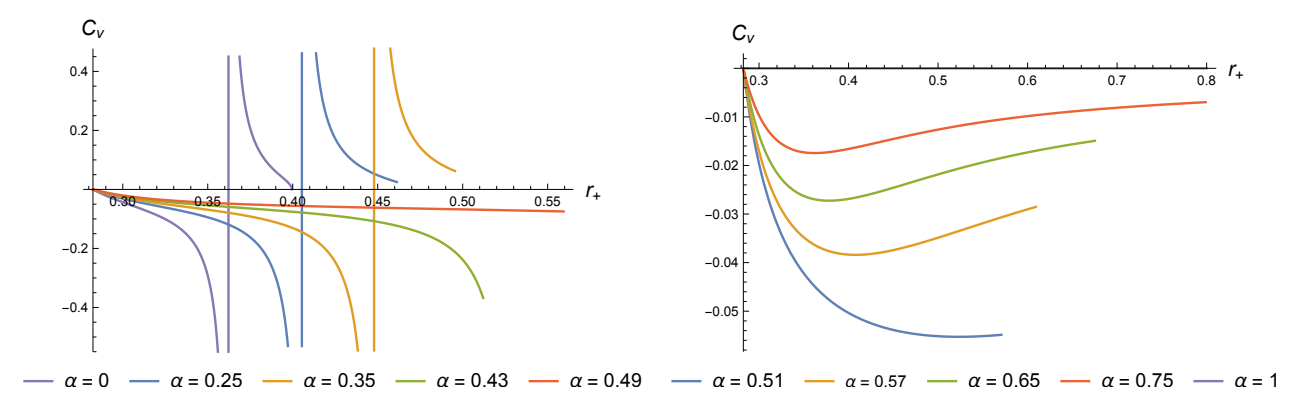

Figure 2. $C_{V}$ of generalized exotic black holes. Left: $\alpha<1 / 2$, right: $\alpha>1 / 2$. In all cases, $P=1, J=0.1$.

\section{Conclusion}

Summarizing, we have shown that generalized exotic BTZ black holes with a sufficiently small $\alpha$ parameter are counter-examples to the conjecture in [1]: they violate the reverse isoperimetric inequality but possess positive $C_{V}$. However we note that an alternate (weaker) interpretation ${ }^{1}$ of the conjecture might be that any connected branch of black holes that is super-entropic will have $C_{V}<0$ for at least some part of the branch and therefore the entire branch is thermodynamically unstable. While our results are consistent with this (weaker) interpretation, we regard it to be less precise since the meaning "connected branch" of black holes is somewhat ambiguous. ${ }^{2}$

We have also observed that $C_{P}<0$ whenever $C_{V}>0$. We therefore posit a further broader conjecture that black holes violating the reverse isoperimetric inequality will be thermodynamically unstable. It will be interesting to see if this broader conjecture can either be proven or if a counterexample can be found.

\section{Acknowledgments}

We would like to thank Antonia Micol Frassino and Jonas Mureika for helpful comments, and Clifford Johnson for helpful correspondence. This work was supported in part by the Natural Sciences and Engineering Research Council of Canada.

Open Access. This article is distributed under the terms of the Creative Commons Attribution License (CC-BY 4.0), which permits any use, distribution and reproduction in any medium, provided the original author(s) and source are credited.

\footnotetext{
${ }^{1}$ We thank Clifford Johnson for pointing this out.

${ }^{2}$ For instance in the charged Schwarzschild-AdS solution, a single temperature can admit two black hole solutions at the same pressure and charge. These belong respectively to "small black hole" and "large black hole" solution branches (see for example [29] for more details). Such distinctions do not occur in the generalized exotic BTZ case [19]. However the divergence in $C_{V}$ can be argued to separate the class of exotic solutions into different branches.
} 


\section{References}

[1] C.V. Johnson, Instability of Super-Entropic Black Holes in Extended Thermodynamics, arXiv: 1906.00993 [INSPIRE].

[2] D. Kubizňák, R.B. Mann and M. Teo, Black hole chemistry: thermodynamics with Lambda, Class. Quant. Grav. 34 (2017) 063001 [arXiv:1608.06147] [INSPIRE].

[3] M. Cvetič, G.W. Gibbons, D. Kubizňák and C.N. Pope, Black Hole Enthalpy and an Entropy Inequality for the Thermodynamic Volume, Phys. Rev. D 84 (2011) 024037 [arXiv: 1012.2888] [INSPIRE].

[4] B.P. Dolan, The cosmological constant and the black hole equation of state, Class. Quant. Grav. 28 (2011) 125020 [arXiv: 1008.5023] [INSPIRE].

[5] D. Kastor, S. Ray and J. Traschen, Enthalpy and the Mechanics of AdS Black Holes, Class. Quant. Grav. 26 (2009) 195011 [arXiv:0904.2765] [INSPIRE].

[6] M.M. Caldarelli, G. Cognola and D. Klemm, Thermodynamics of Kerr-Newman-AdS black holes and conformal field theories, Class. Quant. Grav. 17 (2000) 399 [hep-th/9908022] [INSPIRE].

[7] S. Mbarek and R.B. Mann, Thermodynamic Volume of Cosmological Solitons, Phys. Lett. B 765 (2017) 352 [arXiv: 1611.01131] [INSPIRE].

[8] R.A. Hennigar, D. Kubizňák and R.B. Mann, Entropy Inequality Violations from Ultraspinning Black Holes, Phys. Rev. Lett. 115 (2015) 031101 [arXiv:1411.4309] [InSPIRE].

[9] D. Klemm, Four-dimensional black holes with unusual horizons, Phys. Rev. D 89 (2014) 084007 [arXiv: 1401.3107] [INSPIRE].

[10] R.A. Hennigar, D. Kubizňák, R.B. Mann and N. Musoke, Ultraspinning limits and super-entropic black holes, JHEP 06 (2015) 096 [arXiv: 1504.07529] [INSPIRE].

[11] W.G. Brenna, R.B. Mann and M. Park, Mass and Thermodynamic Volume in Lifshitz Spacetimes, Phys. Rev. D 92 (2015) 044015 [arXiv: 1505.06331] [INSPIRE].

[12] S.M. Noorbakhsh and M. Ghominejad, Ultra-Spinning Gauged Supergravity Black Holes and their Kerr/CFT Correspondence, Phys. Rev. D 95 (2017) 046002 [arXiv:1611.02324] [INSPIRE].

[13] S.M. Noorbakhsh and M. Ghominejad, Higher Dimensional Charged AdS Black Holes at Ultra-spinning Limit and Their 2d CFT Duals, arXiv:1702.03448 [INSPIRE].

[14] X.-H. Feng, H.-S. Liu, W.-T. Lu and H. Lü, Horndeski Gravity and the Violation of Reverse Isoperimetric Inequality, Eur. Phys. J. C 77 (2017) 790 [arXiv:1705.08970] [INSPIRE].

[15] S.M. Noorbakhsh and M.H. Vahidinia, Extremal Vanishing Horizon Kerr-AdS Black Holes at Ultraspinning Limit, JHEP 01 (2018) 042 [arXiv: 1708.08654] [INSPIRE].

[16] R.B. Mann, Super-Entropic Black Holes, Springer Proc. Phys. 208 (2018) 105 [InSPIRE].

[17] M. Bañados, C. Teitelboim and J. Zanelli, The Black hole in three-dimensional space-time, Phys. Rev. Lett. 69 (1992) 1849 [hep-th/9204099] [INSPIRE].

[18] C.V. Johnson, Specific Heats and Schottky Peaks for Black Holes in Extended Thermodynamics, arXiv: 1905.00539 [INSPIRE].

[19] A.M. Frassino, R.B. Mann and J.R. Mureika, Extended Thermodynamics and Complexity in Gravitational Chern-Simons Theory, arXiv:1906.07190 [INSPIRE]. 
[20] A.M. Frassino, R.B. Mann and J.R. Mureika, Lower-Dimensional Black Hole Chemistry, Phys. Rev. D 92 (2015) 124069 [arXiv: 1509.05481] [INSPIRE].

[21] E. Witten, (2+1)-Dimensional Gravity as an Exactly Soluble System, Nucl. Phys. B 311 (1988) 46 [INSPIRE].

[22] P.K. Townsend and B. Zhang, Thermodynamics of "Exotic" Bañados-Teitelboim-Zanelli Black Holes, Phys. Rev. Lett. 110 (2013) 241302 [arXiv:1302.3874] [INSPIRE].

[23] O. Mišković and R. Olea, Background-independent charges in Topologically Massive Gravity, JHEP 12 (2009) 046 [arXiv: 0909.2275] [INSPIRE].

[24] S. Carlip, J. Gegenberg and R.B. Mann, Black holes in three-dimensional topological gravity, Phys. Rev. D 51 (1995) 6854 [gr-qc/9410021] [InSPIRE].

[25] S. Carlip and J. Gegenberg, Gravitating topological matter in $(2+1)$-dimensions, Phys. Rev. D 44 (1991) 424 [INSPIRE].

[26] M. Bañados, Constant curvature black holes, Phys. Rev. D 57 (1998) 1068 [gr-qc/9703040] [INSPIRE].

[27] M. Bañados, A. Gomberoff and C. Martínez, Anti-de Sitter space and black holes, Class. Quant. Grav. 15 (1998) 3575 [hep-th/9805087] [INSPIRE].

[28] S.N. Solodukhin, Holography with gravitational Chern-Simons, Phys. Rev. D 74 (2006) 024015 [hep-th/0509148] [INSPIRE].

[29] D. Kubizňák and R.B. Mann, P - V criticality of charged AdS black holes, JHEP 07 (2012) 033 [arXiv: 1205.0559] [INSPIRE]. 